\title{
Using ipsilateral semitendonosus tendon in reconstruction of infected reruptured patellar tendon in case of avulsed distal patellar pole treated by distal patellar pole excision
}

\begin{abstract}
Background: Infected re-ruptured patellar tendon after avulsed distal patellar pole usually difficult to manage especially if there is bad tissue quality, patella small in size besides atrophy and adhesions of retinacula.

Case presentation: Egyptian male patient 20 years old with re-ruptured repaired patellar tendon after 3 months from management by distal patellar pole excision and repair, (two months in cast and one month physiotherapy) get infected in the ligament site, the infection is treated and reconstruction of the ligament was done using semitendinosus in a figure-ofeight fashion, fixed by sutures and bone tunnels in the patella and tibial tuberosity.
\end{abstract}

Volume 7 Issue I - 2017

\author{
Abdelsamie Halawa,' David Rady² \\ 'Lecturer of orthopedic surgery, Banha medical school, Egypt \\ ${ }^{2}$ Orthopedic specialist, Nasr city insurance hospital, Egypt
}

Correspondence: Abdelsamie Halawa, Lecturer of orthopedic surgery, Banha medical school, Egypt;

Email a_halwah75@yhoo.com

Received: December 27, 2016 | Published: January 05, 2017

Conclusion: The patient get improved and regain near normal extensor mechanism function besides patellar stability and strength.

\section{Introduction}

Fractures of the patella are not uncommon and often result in residual patellofemoral arthralgia, quadriceps weakness, restricted knee motion, and degenerative arthritis. The goals of surgical treatment are to reestablish continuity of the extensor mechanism and to restore a smooth articular surface using stable internal fixation to permit early knee motion. ${ }^{1}$

Despite early conclusions to the contrary, the patella has an important role in both quadriceps and knee joint function. ${ }^{2}$ By increasing the lever arm of the quadriceps mechanism, the patella improves extensor power, particularly during the last $30^{\circ} \circ$ of extension. Maquet's proposal that the patella reduces tibiofemoral contact stresses has been established in experimental animal studies. ${ }^{3}$ Excision of the patella can cause pain, extensor weakness, extensor lag, knee instability, and decreased flexion arc. In one series, like this case - as many as $90 \%$ of patients undergoing patellectomy following fracture had continued pain, and $60 \%$ complained of instability. ${ }^{4}$

In this case the first management was distal patellectomy and reattachment of the patellar tendon. But exposure to truma leads to rerupture again and the problem magnifies. Several reconstruction techniques have been available, including the use of artificial materials; autografts using the semitendinosus, or together with the tendon of gracilis, the contralateral patellar tendon; turndown of the quadriceps tendon; or an allograft using the Achilles tendon. ${ }^{5-8}$ Besides the infection, the technique still difficult to perform because of adhesions, contractures, and atrophy of the quadriceps muscle after surgery. ${ }^{9}$

The idea here is to free the ST tendon graft through small incision over the insertion of pes ansernus, and prepare the two ends using ethbound suture 2 . The tendon the rolled in figure of eight from the tibial tubrosity to the remanant of the patella.

\section{Case presentation}

Male patient 20 years old was exposed to truma lead to avulsion of distal patellar pole Figure 1. The patient underwent management by excision of the distal pole of the patella and direct repair of the tendon to the rest of the patella. Three months later he was exposed to another truma to the knee lead to rerupture of the suture again with tense knee effusion, his seeked medical advice and aspiration was done.

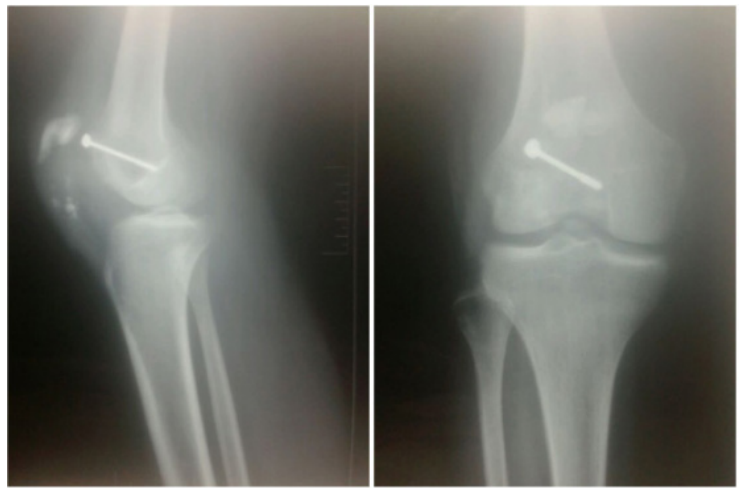

Figure I Preoperaive plane X-rayes shows small sized parella and very high in front of the trochles.

The patient exposed to us by infected knee effusion, lack of extension and knee stiffness. Labs reveals very high ESR and CRP, aggressive parenteral antibacterial was taken for one moth till the labs become negative. During this period ROM exercises was started.

After the passive ROM become complete and labs became negative we plan for him the management, the time lag was 2 months.

\section{The technique}

With the patient supine, under complete aseptic conditions, under umbrella of antibiotics and with midthigh tourniquet, small incision over the pesanserinus insertion was done and the ST tendon was harvested, then prepared and the ends was tapered by ethbound 2 .

Then through midline anterior knee incision, exposure os the patella and tendon was done. Debridement and specimen taken for 
rapid histology and cytology. The edge of the tendon is refreshed and the necrotic tissue excised. Drill hole was done posterior to the tibial tuberosity by a drill pit $4.5 \mathrm{~mm}$ diameter, then tow drill holes was done in the patella from distal to proximal, the tendon then rolled in the tunnel and through patellar tunnels from distal to proximal and again reflected in front of the patella to again the first tunnel in figure of eight manner (Figures $2 \& 3$ ).

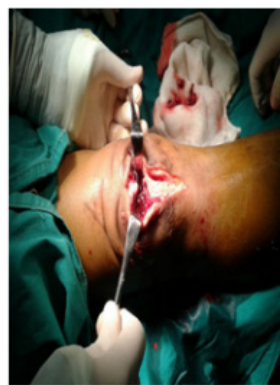

(a)

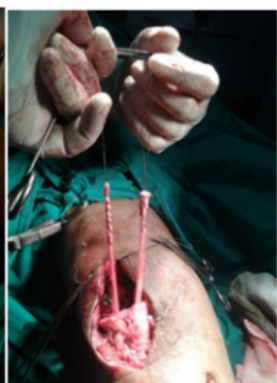

(b)

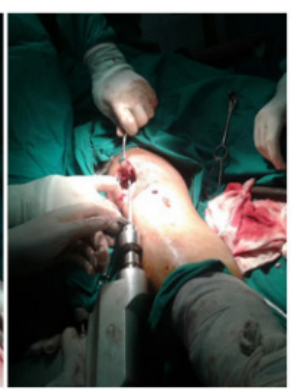

(c)
Figure 2 (a) midline skin incision and debridement then through the same incision tunnel is done and the graft of ST passed though it and the substance (b) of the remanant of the patellar tendon then drilling of the proximal remanant of the patella $(\mathrm{C})$ then pass the tendon through it.

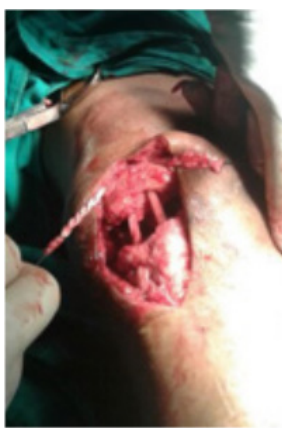

(a)

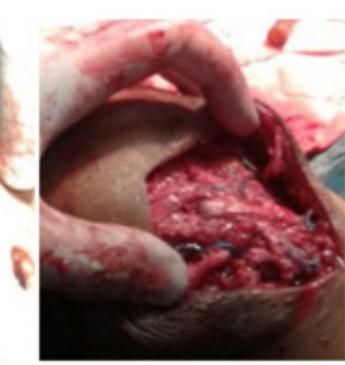

(b)

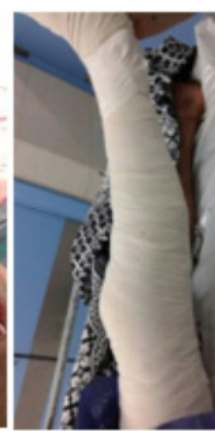

(c)
Figure 3 The tendon is rolled in the Figure of eight (a \& b) and suture the graft on itself in each rolling, The knee is put in pop above knee slab (c).

The tendon was sutured on itself by ethbound sutures, the side retinaculae was approximated and augmentation sutures was taken parallel to the tendon graft. The reconstruction was secured by stainless steel wire Figure 4 all circled around the patella and tibial tunnel. Release was done all sides of the knee to make knee flexion till 90 degrees. Closure in layers over suction drain and will padded pop back knee slab was done.

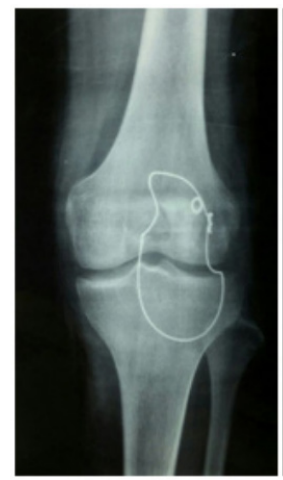

(a)

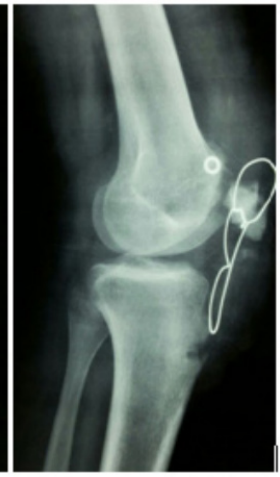

(b)
Figure 4 a $\&$ b Post operative plain X-ray.
The patient then stayed in the hospital for ten days, immediate partial weight bearing and static quadriceps exercises then after 3 weeks started ROM till 30 degree for 3 wks to increase gradually to 90 degrees by 6 weeks, no mentioned complications except the ulcer Figure 5 on the anterior knee skin treated by curettage then 2 nd repair. Now after 6 months the patient can walk freely with mild limping improving by PT and can flex the knee to comfortable range 90 degree Figure 6.

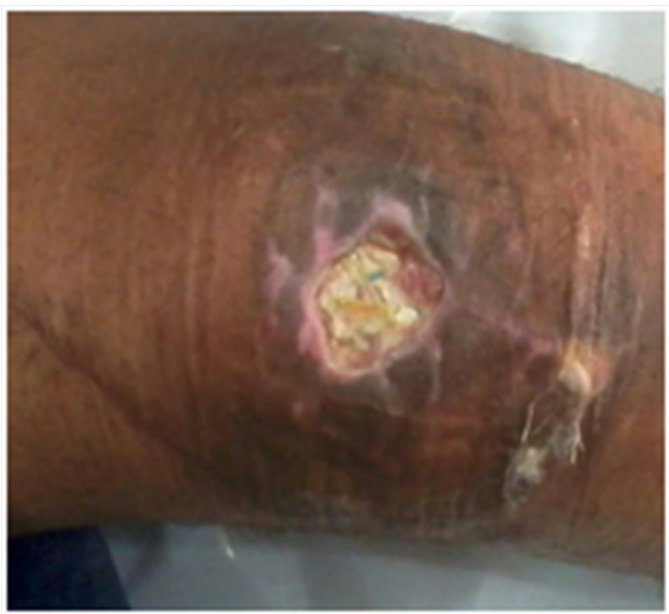

Figure 5 Postoperative skin ulcer.

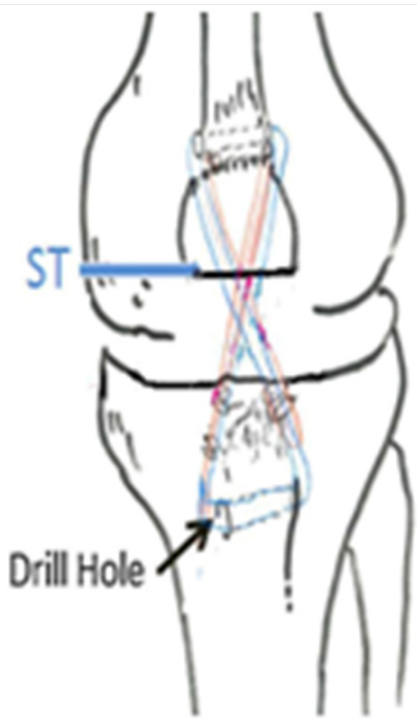

Figure 6 Diagrammatic Figure shows the direction of the semitedonosus and mode of suture.

\section{Discussion}

The patella is a sesamoid bone that lies within the tendon of the quadriceps muscle. Any fracture results in displacement of the fragments in the longitudinal axis represents a disruption of the quadriceps mechanism so a transverse fracture, either simple or comminuted, the quadriceps function is lost and surgical repair is necessary. ${ }^{10}$

Fractures of the patella are one of the fractures that have the ideal indications for tension band fixation with wire beside the olecranon. ${ }^{10}$ If the patella is reduced and held together with a cerclage wire passed circumferentially, when the knee is flexed, the fracture gapes anteriorly, the contour of the patella is changed, and congruence is lost. 
This will also happen as the result of a strong quadriceps contraction, even if the knee is immobilized in extension in a plaster cylinder. With tension band fixation, flexion of the knee results in an increase of compression across the fracture, also contraction of the quadriceps when the knee is extended will not cause the fragments to gape..$^{10}$

Small fragments of the patella, such as an avulsed inferior pole or a lateral fragment, are best fixed to the remainder of the patella with a lag screw. This fixation must be further protected with a tension band wire, if we are obligated to sacrifice the inferior half of the patella because of severe comminution; the infrapatellar tendon is sutured to the remaining bony fragment Figure 7 . The suture line is protected with a tension band wire, which allows early mobilization of the knee. $^{10}$

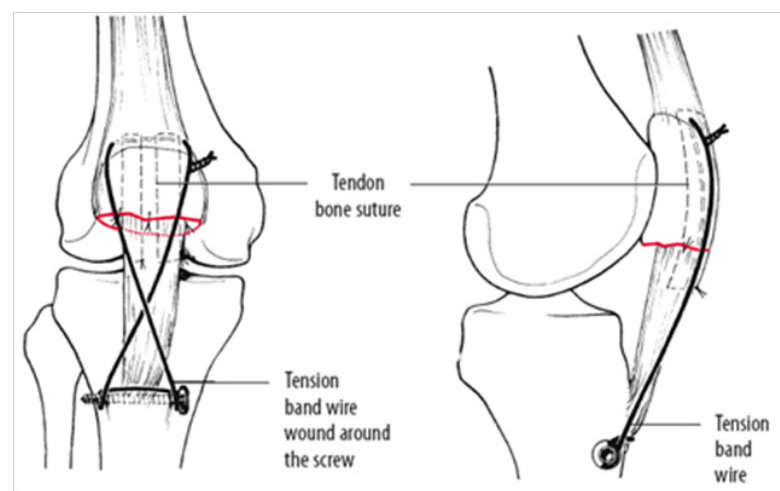

Figure 7 The suture line is protected with a tension band wire, which allows early mobilization of the knee.

In our case the patient was treated by distal pole patellectomy and direct repair by non-absorbable eathbond suture measure 5 , there were no augmentation or security of the repair so the repair failed easily on 2nd trauma.

So in this case we could consider it as rupture of the patellar tendon, which is rare condition. The longer the delay between injury and repair, the greater the likelihood of quadriceps shortening and retraction and proximal patellar migration, fibrous adhesions may develop between the patella and the underlying femur. The ruptured tendon ends shrivel and become encased in scar tissue, reconstruction with extensor mechanism allografts consisting of an Achilles tendon or an intact patellar tendon has also been used as a salvage procedure. ${ }^{11}$

Many techniques have been reported to relocate the patella to its anatomic position and to repair the patellar tendon; however, there is no widely accepted method. ${ }^{11}$

In this case we use all techniques in reconstruction of the patellar tendon as mentioned in the tech. and secure the graft and repair by the crculage wire which will be removed after 1.5 year or when broken, the release of the quadricepse tendon by removing the adhesion between it and the distal femur and also the skin make us easily can bring the patella down.

The three weeks duration in extension posterior knee slab and partial weight bearing protect the graft in relation to the stiffness that could occur as the aim was only get 90 degree flexion in the first 6 months postoperative period, that could be increased later on.
As shown in Figure 8 the degree of flexion of the affected knee in comparison to the normal side after 8 months from the surgery, also the patellar remnant height of the affected side that aids in keeping the function of the patella as a sesamoid bone in the patellar tendon.

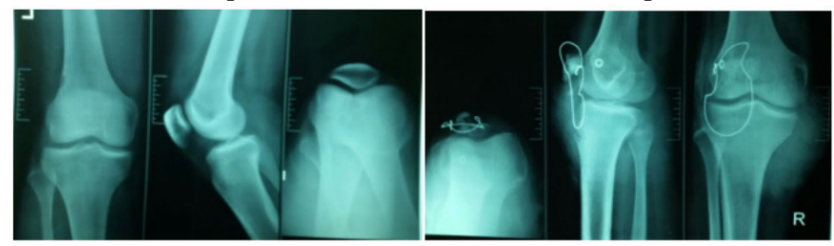

Figure 8 shows the final results after 8 months from the date of surgery and shows the ability of the patient to flex the knee as normal side.

The good functional result that achieved. The positive outcome in this case suggest that this approach should be studied as a possible solution to obtain proper patellar position and functional ROM in cases of infected or neglected patellar tendon rupture.

\section{Acknowledgments}

None.

\section{Conflicts of interest}

None.

\section{References}

1. Timothy $J B 2001$

2. Fractures, Dislocations, Nonunions and Malunions, Patella and Tibia. Chapter 22, Patellar Fractures. Chapman's Orthopaedic Surgery, 3rd edn, Lippincott Williams \& Wilkins, USA.

3. Maquet P Mechanics and Osteoarthritis of the Patellofemoral Joint. Clin Orthop. 1979;144:70-73.

4. Garr EL, Moskowitz RW, Davis W Degenerative Changes Following Experimental Patellectomy in the Rabbit. Clin Orthop. 1973;92:296.

5. Scott JC Fractures of the Patella. J Bone Joint Surg. 1949;31B:76.

6. Kim JR, Park H, Roh SG, Shin SJ Concurrent bilateral patellar tendon rupture in a preadolescent athlete: a case report and review of the literature. J Pediatr Orthop B. 2010;19(6):511-514.

7. Van der Bracht H, Verdonk R, Stuyts B Augmentation of a patellar tendon repair with an autologous graft. Acta Orthop Belg. 2009;75(3):417-419.

8. Milankov MZ, Miljkovic N, Stankovic M Reconstruction of chronic patellar tendon rupture with contralateral BTB autograft: a case report. Knee Surg Sports Traumatol Arthrosc. 2007;15(12):1445-1448.

9. Labib SA, Wilczynski MC, Sweitzer BA Two-layer repair of a chronique patellar tendon rupture: a novel technique and literature review. Am J Orthop. 2010;39(6):277-282.

10. Cadambi A, Engh GA Use of a semitendinosus tendon autogenous graft for rupture of the patellar ligament after total knee arthroplasty. A report of seven cases. J Bone Joint Surg Am. 1992;74(7):974-979.

11. Schatzker J, Marvin T Facture of the patella. Chapter 18, The Rationale of Operative Fracture Care, 3rd edn, pp. 2005;441-446.

12. Burks RT, Edelson RH. Allograft reconstruction of the patellar ligament: a case report. J Bone Joint Surg Am. 1994;76(7):1077-1079. 